\title{
Diabetes mellitus and cardiac complications in thalassemia major patients
}

\author{
Alessia Pepe ${ }^{1 *}$, Antonella Meloni ${ }^{1}$, Vincenzo Caruso ${ }^{2}$, Paolo Cianciulli ${ }^{3}$, Elisabetta Chiodi ${ }^{4}$, Gennaro Restaino $^{6}$, \\ Vincenzo Positano ${ }^{1}$, Petra Keilberg ${ }^{1}$, Massimo Lombardi ${ }^{1}$, Maria Rita Gamberini ${ }^{5}$ \\ From 15th Annual SCMR Scientific Sessions \\ Orlando, FL, USA. 2-5 February 2012
}

\section{Background}

The relationship between diabetes mellitus (DM) and cardiac complications has never been systematically studied in thalassemia major (TM). The aim of this crosssectional study was to evaluate in a large historical cohort of TM in the cardiovascular magnetic resonance (CMR) era if DM was associated with an higher prevalence and risk of heart complications, also regardless to the presence of myocardial iron overload (MIO).

\section{Methods}

We compared 86 TM patients affected by DM with 709 TM patients without DM enrolled in the Myocardial Iron Overload in Thalassemia (MIOT) network at their first CMR. The MIOT network involves 68 thalassemia centers and 8 CMR sites where the exams are performed using standardized and validated procedures and all centers are linked to a web-based database to collect and share patients' history, clinical and diagnostic data.

Cardiac iron was evaluated by $\mathrm{T} 2 *$ multiecho multislice technique. Biventricular function parameters were quantitatively evaluated by cine images. Myocardial fibrosis was evaluated by late gadolinium enhacement CMR technique.

Heart failure (HF) was diagnosed by CMR in presence of a left ventricular (LV) and/or right ventricular (RV) ejection fraction (EF) lower than 4 standard deviations from the mean value normalized by sex and age. Moreover HF was identified by patients' history, diagnosed by clinicians based on symptoms, signs and echocardiographic findings. All considered cardiac events were developed after the DM diagnosis.

${ }^{1}$ CMR Unit, Fondazione G.Monasterio CNR-Regione Toscana and Institute of Clinical Physiology, Pisa, Italy

Full list of author information is available at the end of the article

\section{Results}

In DM patients versus no-DM patients we found a significantly higher prevalence of cardiac complications (HF, arrhythmias and pulmonary hypertension) (46.5\% vs $16.9 \%, \mathrm{P}<0.0001)$, $\operatorname{HF}(30.2 \%$ vs $11.7 \%, \mathrm{P}<0.0001)$, hyperkinetic arrhythmias ECG documented and requiring medications $(18.6 \%$ vs $5.5 \%, \mathrm{P}<0.0001)$, and myocardial fibrosis (29.9\% vs $18.4 \%, \mathrm{P}=0.008)$.

To evaluate the impact of the DM on cardiac complications also in relationship to MIO, we always adjusted the risk of cardiac findings for the absence of MIO (all segments with $\mathrm{T}_{2} * 20 \mathrm{~ms}$ ). Patients with $\mathrm{DM}$ had a significant higher risk of cardiac complications (OR 2.84, $\mathrm{P}<0.0001)$, HF (OR 2.32, $\mathrm{P}=0.003)$, hyperkinetic arrhythmias (OR 2.21, $\mathrm{P}=0.023$ ) and myocardial fibrosis (OR 1.91, $\mathrm{P}=0.021)$.

\section{Conclusions}

DM increases the risk for cardiac complications, HF, hyperkinetic arrhythmias and myocardial fibrosis irrespective of MIO.

\section{Funding}

"No-profit" support by industrial sponsorships (Chiesi, Apotex and GE Healtcare) and "Ministero della Salute, fondi ex art. 12 D.Lgs. 502/92 e s.m.i., ricerca sanitaria finalizzata anno 2006" e "Fondazione L. Giambrone".

\section{Author details \\ ${ }^{1}$ CMR Unit, Fondazione G.Monasterio CNR-Regione Toscana and Institute of Clinical Physiology, Pisa, Italy. ${ }^{2}$ Unità Operativa Dipartimentale Talassemia, P. O. "S. Luigi-Currò" - ARNAS Garibaldi, Catania, Italy. ${ }^{3}$ Centro Talassemie, Ospedale "Sant'Eugenio Papa", Roma, Italy. "Dipartimento di Radiologia, Ospedale "Sant'Anna", Ferrara, Italy. ${ }^{5}$ Pediatria, Adolescentologia e Talassemia, Ospedale "Sant'Anna", Ferrara, Italy. 'Departement of radiology, "John Paul II" Catholic University, Campobasso, Italy.}

Published: 1 February 2012 
Table 1 Logistic regression analysis: ORs (95\% CI) of DM versus no-DM patients for cardiac findings adjusted for the covariates significantly different between groups and significantly associated to the dependent variable.

\begin{tabular}{|c|c|c|c|c|}
\hline & \multicolumn{2}{|c|}{ Adjusted for no-MIO } & \multicolumn{2}{|c|}{ Adjusted for no-MIO and covariates } \\
\hline & OR $(95 \% \mathrm{Cl})$ & P-value & OR $(95 \% \mathrm{Cl})$ & P-value \\
\hline Cardiac complications & $4.23(2.65-6.76)$ & $<0.0001$ & $2.84(1.71-4.69) \#$ & $<0.0001$ \\
\hline Heart failure & $3.14(1.87-5.26)$ & $<0.0001$ & $2.33(1.33-4.06) \#$ & 0.003 \\
\hline Heart failure at time of study & $3.45(2.02-5.89)$ & $<0.0001$ & $2.48(1.39-4.43) \#$ & 0.002 \\
\hline Hyperkinetic arrhythmias & $4.09(2.16-7.74)$ & $<0.0001$ & $2.21(1.12-4.37) \#$ & 0.023 \\
\hline Myocardial fibrosis & $2.12(1.24-3.63)$ & 0.006 & $1.91(1.11-3.29) \S$ & 0.021 \\
\hline Heart dysfunction (LV and/or RV) & $1.45(0.37-2.33)$ & 0.093 & & \\
\hline Biventricular dysfunction & $1.46(0.78-2.72)$ & 0.235 & & \\
\hline LV dysfunction & $0.77(0.37-1.60)$ & 0.487 & $1.62(0.44-5.97) *$ & 0.470 \\
\hline RV dysfunction & $1.82(1.01-3.30)$ & 0.048 & $1.33(0.71-2.49) *$ & 0.366 \\
\hline
\end{tabular}

Covariates: * age; $\S$ endocrine co-morbidity; \# age and endocrine co-morbidity.

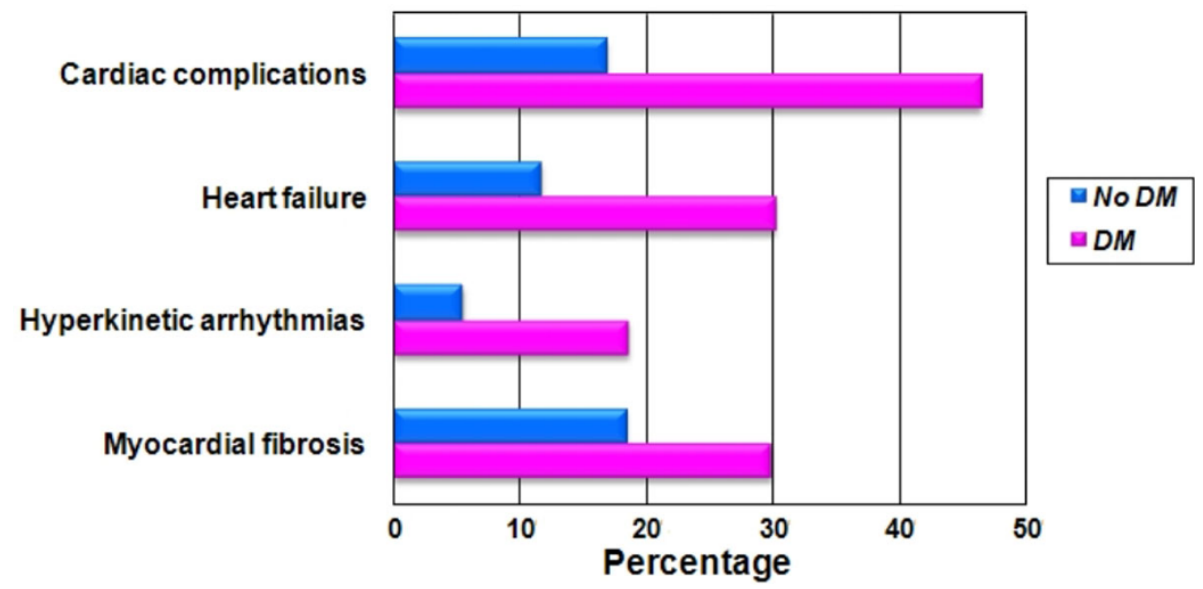

Figure 1 Prevalence of significant cardiac end-points for patients with and without DM.

Submit your next manuscript to BioMed Central and take full advantage of:

- Convenient online submission

- Thorough peer review

- No space constraints or color figure charges

- Immediate publication on acceptance

- Inclusion in PubMed, CAS, Scopus and Google Scholar

- Research which is freely available for redistribution 